\title{
Mobile Learning: aproximación conceptual y prácticas colaborativas emergentes
}

UT. Revista de Ciències de l'Educació

Desembre 2011. Pag. 43-50

ISSN 1135-1438

http://pedagogia.fcep.urv.cat/revistaut

\author{
Mar Camacho Martí \\ Rebut: 30/11/2011 Acceptat: 12/12/2011
}

\section{Resumen}

La fuerte irrupción de los dispositivos móviles y los medios de comunicación social en nuestra vida cotidiana ha transformado no sólo el modo en que nos comunicamos y relacionamos, sino también el modo en que aprendemos y enseñamos. Las tecnologías móviles han cambiado la propia naturaleza del conocimiento, la forma en que se distribuye y cómo se accede a él. El uso extendido por parte de los jóvenes de teléfonos móviles (según datos del año 2010 del Instituto de la Juventud, el 93\% de los jóvenes españoles dispone de un teléfono móvil) reproductores, tablets, consolas y todo tipo de dispositivos móviles dibuja un panorama en el que es posible crear y acceder a nuevas formas de conocimiento, y evidencia a la vez la necesidad de afianzar una base conceptual sólida sobre la que fundamentar su desarrollo. El objetivo de este artículo tiene una doble vertiente, por una parte explorar el concepto y definición de Mobile Learning así como sus potencialidades educativas. Por otra, evidenciar dicho potencial cuando éste es extrapolado a acciones colaborativas y de generación de conocimiento compartido, como es el caso del proyecto que se presenta: The icollab project, un proyecto de colaboración internacional basado en el uso de tecnologías móviles y herramientas web 2.0 para la creación y el mantenimiento de una comunidad virtual de práctica entre diferentes profesores y alumnado de diferentes partes del mundo.

Palabras Clave: Mobile_learning, Web 2.0, colaboración, aprendizaje, redes_sociales

\footnotetext{
${ }^{a}$ Departament de Pedagogia. Universitat Rovira i Virgili - Tarragona
} 


\section{Mobile Learning: conceptual approach and emerging collaborative practices}

\section{Abstract}

The strong emergence of mobile devices and social media in our daily lives has affected not only the way in which we communicate and interact with others, but also how we learn and teach. Mobile technologies have changed the nature of knowledge, the way it is distributed and how it is accessed. The widespread use by young mobile phone (according to the Instituto de la Juventud in 2010) 93\% of Spanish teenagers people have a mobile phone) players, tablets, consoles and mobile devices of all kinds, draws a picture where it is possible to create and access new forms of knowledge, at the time that evidences the need to strengthen a sound conceptual basis on which to base their development. The aim of this paper is twofold, firstly it aims to to explore the concept and definition of mobile learning and its educational potential. Seccondly, it aims at evidencing this potential when it is extrapolated to collaborative action and shared knowledge generation, as is the case of the project presented: The icollab project, a project of international cooperation based on the use of mobile technologies and web 2.0 tools for creating and maintaining a virtual community of practice among different teachers and pupils from different parts of the world

Key Words: Mobile_learning, Web 2.0, collaboration, learning, social networks

\section{Hacia una definición clara de Mobile learning}

La literatura existente sobre Mobile Learning ha ido evolucionando en los últimos años para desplazarse desde una visión absolutamente tecno-céntrica a otra percepción mucho más educativa (Buchem, Camacho: 2011) que entiende Mobile learning como "aprendizaje con dispositivos móbiles" Así, Traxler (2005) describe Mobile Learning como cualquier acción educativa donde las tecnologías dominantes son móviles" y Quinn (2000) como "un tipo de e-learning a través de dispositivos móviles". Sin embargo, O'Malley et al. (2003) y otros autores como Keegan (2005) describen Mobile Learning como "aquél aprendizaje que tiene lugar cuando el estudiante no se encuentra en un lugar determinado o fijo" o bien como "el aprendizaje que tiene lugar cuando el estudiante se beneficia de las oportunidades de aprendizaje ofrecidas por las tecnologías móviles". Finalmente, la iniciativa MoLeNET (2009) describe Mobile Learning como "la explotación de tecnologías ubicuas de mano, juntamente con redes para facilitar, apoyar, mejorar y ampliar el alcance de la enseñanza y el aprendizaje".

Aunque los beneficios de utilizar este tipo de aprendizaje hayan sido subrayados por numerosos autores (Laurillard, 2007, Pacheco, Bachmair \& Cook, 2010 .. ) es cada vez más difícil ubicar el Mobile Learning dentro de las constricciones de la educación formal si no se basa en algún tipo de racional que permita construir una consostente 
teoría de aprendizaje. Así, Mobile Learning posee diferentes formas dibujadas en una serie de diferentes teorías y métodos pedagógicos. Desde este punto de vista, es difícil especificar un único racional y necesario detallar, como señalan varios autores, diferentes percepciones metodológicas y aplicaciones prácticas que se engloban bajo el término (Buchem, Camacho, 2011, Frohberg, Goth y Schwabe, 2009). Así, podemos hablar de Mobile Learning para la distribución de contenidos (Bradley et al, 2009; Muyinda, Lubega \& Lynch 2010), como facilitador de procesos reflexivos, (Corlett et al, 2005.), como base para desarrollar e implementar juegos móviles basados en el aprendizaje (Mitchell, Millwood y Fallenboeck, 2006). Autores como Cochrane y Bateman, 2010;. Safran et al, 2010) hablan de una Web 2.0 móvil, aunque cabe destacar y hacer hincapié en el hecho que el beneficio del aprendizaje móvil está dado por la portabilidad, flexibilidad y el contexto de las tecnologías móviles, lo que permite el aprendizaje, promover la colaboración y fomentar el aprendizaje autónomo para toda la vida (Naismith et al, 2004 Traxler, 2009, Dyson et al, 2008).

Otros autores han destacado también el potencial del Mobile Learning para el desarrollo de las competencias genéricas necesarias para el éxito de la educación y el desarrollo profesional (Litchfield, Nettleton y Taylor, 2008) y han evidenciado la necesidad de integrarlo en la práctica educativa y la enseñanza superior (Traxler, 2005; Dyson, Raban, Litchfield y Lawrence, 2008). Llegados a este punto, consideramos conveniente destacar la relación que se establece entre las teorías del aprendizaje constructivo y el Mobile Learning, puesto que éste propicia que se dé una interacción social para la co-construcción de conocimiento a la vez que permite al estudiante construir andamiajes para el aprendizaje significativo. Es importante también enfatizar la idea del estudiante que lleva control de su aprendizaje (creando sus propios objetivos y procesos) colaborando con sus pares para producir contenido y utilizar sus dispositivos móviles para la investigación y exploración (Loke et al. 2010).

\section{Mobile Learning en educación}

El uso de Mobile Learning en procesos de enseñanza y aprendizaje sea al nivel educativo que sea, nos ofrece retos para desarrollar nuevos enfoques pedagógicos centrados en el potencial de los dispositivos móviles ya sea en contextos formales o informales. Pensamos que es importante siempre tener en cuenta que Mobile Learning no es sólo aprendizaje con las tecnologías móviles, sino que también se considera un nuevo tipo de aprendizaje. Finalmente, el aprendizaje móvil cruza el límite de aprendizaje institucional (intersección ámbito formal-informal) y plantea la necesidad de generar - por parte tanto de instituciones como agentes involucradosespacios compartidos de generación e intercambio de conocimientos que den cabida a este "otro" pero también válido tipo de aprendizaje.

Entre las muchas características de Mobile Learning en educación destacar: las sigüientes: 
- $\quad$ aprendizaje centrado en el entorno y contexto del estudiante

- $\quad$ permite la publicación directa de contenidos, observaciones y reflexiones

- favorece la interacción y la colaboración

- facilita la creación de comunidades de aprendizaje

- permite que las nuevas habilidades o conocimientos se apliquen inmediatamente

- $\quad$ enfatiza el aprendizaje auto-dirigido y diferenciado

- $\quad$ ofrece posibilidades de capturar fácilmente momentos irrepetibles sobre los cuales hacer debate y reflexión

- favorece la colaboración distribuida y numerosas oportunidades de trabajo

Por otra parte cabe destacar la gran idoneidad de utilizar los dispositivos móviles en proyectos colaborativos como destacan diversos estudios relacionados también con la utilización de diferentes herramientas de la Web 2.0 para promover y potenciar la dimensión comunicativa del alumnado así como la vertiente creativa en lo que a contenidos se refiere. Así, es necesario hacer referencia a los estudios que valoran:

- El potencial del M-Learning para la colaboración internacional, (A. Gordon y Picherit Duther, 2009; AE Gordon, 2011)

- El uso de Google Docs para el mapeo de los proyectos de colaboración y gestión de los profesores,

- la utilización de Twitter para la comunicación y el intercambio de ideas (Buchem, 2011; Cochrane, 2010).

- La creación de blogs de los estudiantes para mantener al día el progreso del aprendizaje (Cochrane y Bateman, 2011)

- La generación de contenidos desde móviles a partir de imágenes georeferenciadas compartidas a través de Flickr o Picasaweb, y el vídeo compartido a través de YouTube) (Keegan, 2010)

- La utilización de Prezi presentado reunión y la participación crítica de los estudiantes (Buchem \& Camacho, 2011)

- El uso de la realidad aumentada para representar en diferentes capas la realidad (por ejemplo, mediante enlaces a imágenes de arquitectura, QRCodes, episodios móviles de vídeo en YouTube..) (Cochrane y Rhodes, 2011) 
Mobile Learning: aproximación conceptual y prácticas colaborativas emergentes

- Uso de Skype o Google Plus Hangout y vodcasts para presentaciones remotas

\section{Mobile learning en procesos colaborativos: The icollab project}

La proliferación de numerosas herramientas sociales que permiten diferentes tipos de interacción y comunicación, la generación y publicación inmediata de contenidos y la aparición de nuevas formas de expresión, de nuevos lenguajes y narrativas hace necesaria una reconceptualización de aproximaciones metodológicas que hay que tener en cuenta y saber incorporar en los procesos de enseñanza y aprendizaje. De esta misma manera, se hace necesario explorar los cambios que el uso reiterado de las pantallas pueden provocar en los hábitos lectores, las formas de escritura y los lenguajes narrativos y proporcionar mecanismos y estrategias de colaboración que puedan dar respuesta a las diferentes necesidades tanto de alumnado como profesorado.

Es bajo este marco donde se ubica The Icollab project, proyecto de colaboración internacional basado en el uso de herramientas de la Web 2.0 y el Mobile learning, iniciado en 2011 en el que participamos desde el grupo de investigación ARGET del Departamento de Pedagogía. Se trata de un proyecto de colaboración internacional emergente cuyo objetivo básico es el de utilizar de manera colaborativa tecnologías móviles y herramientas web 2.0 para la facilitación y el mantenimiento de una comunidad virtual de práctica entre diferentes profesores y alumnado, pertenecientes a diferentes partes del mundo. Los participantes en este proyecto son estudiantes y profesores de las sigüientes universidades: AUT University (Nueva Zelanda), Sheffield University and Salford University (Reino Unido), Beuth University of Applied Sciences Berlin (Alemania) y la Universitat Rovira i Virgili

Si bien se trata de un proyecto que se encuentra en sus inicios, cabe destacar que en el proceso de negociación y planificación del proyecto los profesores han experimentado con diferentes herramientas de la Web 2.0 con fines colaborativos de las que ya se ha apropiado los alumnos para la formación de equipos de colaboración y también para la realización de diferentes tareas académicas. .El proyecto trata de salvar los contextos de aprendizaje más allá de los contextos locales de aprendizaje formal e informal así como potenciar la colaboración internacional. Tanto los dispositivos móviles como las herramientas de la Web 2.0 se han convertido en facilitadores esencialmente sociales y móviles de la colaboración internacional en este proyecto. El contacto inicial con varios de los miembros del proyecto ha sido totalmente a través de móviles de redes sociales como Twitter, junto con Google y Youtube. El establecimiento alianzas internacionales presenta una oportunidad emocionante para fomentar estrategias de colaboración y profundizar a la vez en mecanismos y estrategias de aprendizaje y construcción de conocimiento compartido. El proyecto también constituye un catalizador interesante para la introducción de la transformación pedagógica del docente centrada en un curriculum dirigido a estudiantes donde los diferentes proyectos llevados a cabo son negociados 
previamente (heutagogy). Los resultados del proyecto y la retroalimentación ofrecida por parte de los estudiantes será la base para la creación de nuevas líneas de trabajo enmarcadas en el ámbito específico que ofrece el Mobile Learning.

\section{Conclusiones}

La definición y alcance de Mobile Learning es fundamental para el debate sobre el uso pedagógico de los dispositivos móviles. El uso de los teléfonos móviles y otros dispositivos cada vez más ejercen una influencia no sólo sobre los paradigmas del aprendizaje, sino también en el repertorio de agentes y parámetros relacionados con éste. Si bien es cierto que el uso de los dispositivos móviles presenta una gran oportunidad y ofrece un desafío para redefinir y transformar nuestros paradigmas educativos, el aprendizaje móvil también requiere de una nueva filosofía marco que le acoja entre los nuevos paradigmas educativos y unifique sus potencialidades. En el caso de los proyectos de colaboración Mobile Learning ofrece a los agentes involucrados en los diferentes procesos educativos un panorama lleno de gran potencial, parte del cual está aún por explorar. Proyectos colaborativos basados en el uso de dispositivos móviles y vinculados al uso educativo de las herramientas de la Web 2.0 como es el caso de The Icollab Project son fundamentales y deben servir de catalizadores de cambio para la introducción del Mobile Learning en el ámbito más educativo, a la vez que favorecen -por parte del alumnado- la generación de contenidos y el intercambio constante de experiencias culturales y también sociales.

\section{Referencias}

BUCHEM, I., CAMACHO, M., (2011) M-project: first steps to Applying Action Research in Designing a Mobile Learning Course in Higher Education. In Rummler, $\mathrm{K}$, Seipold,J,Lübcke,E,Pachler,N, Attwell,G (2011) Mobile learning: Crossing boundaries in convergent environments. London Mobile Learning Group, Germany ISSN 1753-3385, pages: $123-132$.

Corlett, D., Chan, T., Ting, J., Sharples, M., \& Westmancott, O. (2005) Interactive Logbook: a Mobile Portfolio and Personal Development Planning Tool. In H. van der Merwe \& T. Brown, Mobile Technology.

Cochrane, T., \& Bateman, R. (2010). Smartphones give you wings: Pedagogical affordances of mobile web 2.0. Australasian Journal of Educational Technology, 26(1), pp. 1-14.

Delfino, M., Dettori, G. and Lupi, V. (2009). Task-based learning and ICT: creative activities in the context of a European project. elearning Papers No. 16.

Dyson, L. E., Litchfield, A., Lawrence, E., Raban, R. \& Leijdekkers, P. (2009). Advancing the $\mathrm{m}$-learning research agenda for active, experiential learning: Four case studies. Australasian Journal of Educational Technology, 25(2), 250-267. 
Frohberg, C. Göth, \& Schwabe G. (2009). Mobile Learning projects -a critical analysis of the state of the art, Journal of Computer Assisted Learning, 25 (4), pp. 307-331

Jonassen, D.H. (1991). Evaluating Constructivist Learning. Educational Technology, 31(9), pp. 28-33.

Lefoe, G., Olney, I., Wright, R. \& Herrington, A. (2009). Faculty development for new technologies: Putting mobile learning in the hands of the teachers. In J. Herrington, A. Herrington, J. Mantei, I. Olney, \& B. Ferry (Eds.), New technologies, new pedagogies: Mobile learning in higher education (pp. 15-27). Wollongong: University of Wollongong.

Mcloughlin, C \& Lee M. J. W. (2007) Social software and participatory learning: Pedagogical choices with technology affordances in the Web 2.0 era. ascilite 2007. Singapore.

Mitchell, A., Millwood, R. \& Fallenboeck, M. (2006). Towards a pedagogical framework for the mobile Game-Based Learning project - key considerations. Conference ICL2006 2006 Villach, Austria, 1(9)

Molenet (2009). The Impact of mobile learning https://crm.Isnlearning.org.uk/user/order.aspx?code=090068

Sharples, M. (2005). Learning as conversation: Transforming education in the mobile age. Proceedings Seeing Understanding, Learning in the Mobile Age, Budapest, April 28-30, 2005, pp. 147-152.

The Future of Learning in Your Hands, mLearn 2005 Book of Abstracts, 4th World Conference on mLearning, Cape Town, 25-28 October 2005. Cape Town: mLearn 2005, pp. 22.

Wingkvist, A. (2008). The Quest for Equilibrium: Towards an Understanding of Scalability and Sustainability for Mobile Learning. Licentiate thesis. Växjö University.

\section{Nota biográfica}

Mar Camacho, es docente e investigadora en el Departamento de Pedagogía de la Facultad de Educación de la Universitat Rovira i Virgili (Tarragona - Cataluña) y miembro desde 2001 del grupo de Investigación de Tecnología Educativa de la misma universidad ARGET. De 2004-2009 trabajó como consultora en la Dirección General de Innovación del Departament d' Educació de la Generalitat de Catalunya. En la actualidad imparte clases y cursos en diferentes programas de Master, Doctorado y Postgrado relacionados con la Tecnología Educativa. Autora de diversas publicaciones sobre el uso de las TIC en la enseñanza de idiomas y los procesos de aprendizaje, el uso de la Web 2.0 y sus herramientas y recursos para la docencia, especialmente en contextos multiculturales y plurilingües y sobre el uso y aplicación 
del Mobile Learning y las tecnologías emergentes como herramientas que nos ayudan a transformar, enriquecer y ampliar la experiencia del aprender.

Durante los últimos años ha estado trabajando en proyectos de investigación sobre metodologías docentes y las TIC, el diseño y desarrollo de cursos de formación del profesorado y, básicamente, en el uso de herramientas móviles destinadas a mejorar los procesos de aprendizaje, incluyendo el uso de podcasts como herramienta para facilitar la adquisición de la competencia oral y comunicativa para promover la diversidad en la Europa multicultural. Actualmente se encuentra trabajando en diferentes proyectos que se desarrollan en el marco del Mobile Learning y otras tecnologías emergentes como el Game-based learning y el aprendizaje en metaversos y mundos 3D. En los últimos años ha participado activamente en seminarios, mesas redondas y conferencias como ONLINE EDUCA BERLIN, ED-MEDIA, EDUTEC, MOBILE IADIS, EDEN, ECER, PLE CONFERENCE, E-CHALLENGES, SCOPEO ... 